\title{
ANALYTIC INTEGRABILITY OF HAMILTONIAN SYSTEMS WITH A HOMOGENEOUS POLYNOMIAL POTENTIAL OF DEGREE 4
}

\author{
JAUME LLIBRE $^{1}$, ADAM MAHDI $^{2,3}$ AND CLAUDIA VALLS $^{4}$
}

\begin{abstract}
In the analytic case we prove the conjecture of A.J. Maciejewski and M. Przybylska that appeared in J. Math. Phys. 46 (6) (2005) 062901 regarding Hamiltonian systems with a homogeneous polynomial potential of degree 4 . The proof of the conjecture completes the characterization of all the analytic integrable Hamiltonian system with a homogeneous polynomial potential of degree 4 .
\end{abstract}

\section{IntRoduCtion AND STATEMENT OF THE MAIN RESUlT}

We consider $\mathbb{C}^{4}$ as a symplectic linear space with canonical variables $\mathbf{q}=\left(q_{1}, q_{2}\right)$ and $\mathbf{p}=\left(p_{1}, p_{2}\right)$. We are interested in Hamiltonian systems defined by the Hamiltonian function

$$
H=\frac{1}{2} \sum_{i=1}^{2} p_{i}^{2}+V(\mathbf{q}),
$$

where $V(\mathbf{q})=V\left(q_{1}, q_{2}\right)$ is a homogeneous polynomial of degree $k$. To be more precise we consider the following system of four differential equations

$$
\dot{q}_{i}=p_{i}, \quad \dot{p}_{i}=-\frac{\partial V}{\partial q_{i}}, \quad i=1,2 .
$$

Let $A=A(\mathbf{q}, \mathbf{p})$ and $B=B(\mathbf{q}, \mathbf{p})$ be two functions. Then their Poisson bracket $\{A, B\}$ is given by

$$
\{A, B\}=\sum_{i=1}^{2}\left(\frac{\partial A}{\partial q_{i}} \frac{\partial B}{\partial p_{i}}-\frac{\partial A}{\partial p_{i}} \frac{\partial B}{\partial q_{i}}\right) .
$$

We say that functions $A$ and $B$ commute or that they are in involution if $\{A, B\}=0$. We say that a non-constant function $F=F(\mathbf{q}, \mathbf{p})$ is a first integral for the Hamiltonian system (2) if it commutes with the Hamiltonian function $H$, i.e. $\{H, F\}=0$. Since the Poisson bracket is antisymmetric it is clear that $H$ itself is always a first integral. We will say that a 2 -degree of freedom Hamiltonian system (2) is completely or Liouville integrable if it has 2 functionally independent first integrals: $H$, and an additional one $F$, which are in involution.

In the beginning of 80's all integrable Hamiltonian systems (1) with homogeneous polynomial potential of degree at most 5 and having a second polynomial first integral up to degree 4 in the variables $p_{1}$ and $p_{2}$ were found, see $[11,4,2,5,1]$ and also [6] for the list of corresponding additional first integrals. We remark that all these first integrals are polynomials in the variables $p_{1}, p_{2}, q_{1}$ and $q_{2}$. The main tools used there in order to identify these integrable systems were Panlevé test [3] and direct methods [7].

Key words and phrases. Analytic integrability, Hamiltonian system with 2-degrees of freedom, homogeneous polynomial potential. 
An elegant result related with the integrability of Hamiltonian systems with a homogeneous polynomial potential was given by Morales and Ramis (see [10, p. 100] and references therein), which gives the necessary condition for the complete meromorphic integrability of such systems.

Using the result of Morales-Ramis, Maciejewski and Przybylska [8] gave a necessary and sufficient condition for the complete meromorphic integrability of Hamiltonian systems with the homogeneous polynomial potential of degree 3. Later on in [9] the same authors studied, among other things, the meromorphic integrability of the class of Hamiltonian systems with a homogeneous polynomial potential of degree 4 . They proved that except for one family of potentials, only those systems which are already known to be integrable with a polynomial first integral, are all the meromorphically integrable systems. For this exceptional family they state:

Conjecture ([9]). The Hamiltonian system (2) with the potential

$$
V=\frac{1}{2} a q_{1}^{2}\left(q_{1}+i q_{2}\right)^{2}+\frac{1}{4}\left(q_{1}^{2}+q_{2}^{2}\right)^{2}
$$

is meromorphically integrable if and only if $a=0,-2$.

For $a=0$ and $a=-2$ the additional first integrals are given in Table 1 . The case $a=0$ is a special case of the following more general result. The Hamiltonian system (1) having a potential $V=f\left(q_{1}^{2}+q_{2}^{2}\right)$ is integrable with the additional first integral $I=p_{1} q_{2}-p_{2} q_{1}$.

\begin{tabular}{|l|l|l|}
\hline Case & Potential V & Additional first integral \\
\hline$a=0$ & $\frac{1}{4}\left(q_{1}^{2}+q_{2}^{2}\right)^{2}$ & $p_{1} q_{2}-p_{2} q_{1}$ \\
$a=-2$ & $-q_{1}^{2}\left(q_{1}+i q_{2}\right)^{2}+\frac{1}{4}\left(q_{1}^{2}+q_{2}^{2}\right)^{2}$ & $p_{1}^{2}+3 i p_{1} p_{2}-2 p_{2}^{2}+\left(q_{1} i-q_{2}\right)^{3} q_{2}$ \\
\hline
\end{tabular}

TABLE 1. Two integrable cases of the potential (3) and the corresponding additional first integrals.

The main purpose of this article is to prove the conjecture in the analytic case, i.e.:

Theorem 1. The Hamiltonian system (2) with the potential (3) is analytically integrable if and only if $a=0,-2$.

In short Theorem 1 completes the characterization of all analytic Hamiltonian systems (2) having a homogeneous polynomial potential of degree 4 .

The way of proving Theorem 1 is to show that such Hamiltonian systems have no additional analytic first integrals when $a \neq 0,-2$. In section 2 we shall see that our differential system is weight-homogeneous. This fact will be used in section 3 for proving Theorem 1 .

\section{WeIGHT-HOMOGENEOUS POLYNOMIAL DIFFERENTIAL SYSTEM}

We consider polynomial differential systems of the form

$$
\frac{d \mathbf{x}}{d t}=\dot{\mathbf{x}}=\mathbf{P}(\mathbf{x}), \quad \mathbf{x}=\left(x_{1}, x_{2}, x_{3}, x_{4}\right) \in \mathbb{C}^{4},
$$

with $\mathbf{P}(\mathbf{x})=\left(P_{1}(\mathbf{x}), P_{2}(\mathbf{x}), P_{3}(\mathbf{x}), P_{4}(\mathbf{x})\right)$ and $P_{i} \in \mathbb{C}\left[x_{1}, x_{2}, x_{3}, x_{4}\right]$ for $i=1,2,3,4$. As usual $\mathbb{N}, \mathbb{R}$ and $\mathbb{C}$ denote the sets of positive integers, real and complex numbers, respectively; and $\mathbb{C}\left[x_{1}, x_{2}, x_{3}, x_{4}\right]$ denotes the polynomial ring over $\mathbb{C}$ in the variables $x_{1}, x_{2}, x_{3}, x_{4}$. Here $t$ can be real or complex. 
We say that system (4) is weight-homogeneous if there exist $\mathbf{s}=\left(s_{1}, s_{2}, s_{3}\right.$, $\left.s_{4}\right) \in \mathbb{N}^{4}$ and $d \in \mathbb{N}$ such that for arbitrary $\alpha \in \mathbb{R}^{+}=\{\alpha \in \mathbb{R}, \alpha>0\}$,

$$
P_{i}\left(\alpha^{s_{1}} x_{1}, \alpha^{s_{2}} x_{2}, \alpha^{s_{3}} x_{3}, \alpha^{s_{4}} x_{4}\right)=\alpha^{s_{i}-1+d} P_{i}\left(x_{1}, x_{2}, x_{3}, x_{4}\right),
$$

for $i=1,2,3,4$. We call $\mathbf{s}=\left(s_{1}, s_{2}, s_{3}, s_{4}\right)$ the weight exponent of system (4) and $d$ the weight degree with respect to the weight exponent $\mathbf{s}$. In the particular case that $\mathbf{s}=(1,1,1,1)$ system (4) is called a homogeneous polynomial differential system of degree $d$.

We say that a polynomial $F\left(x_{1}, x_{2}, x_{3}, x_{4}\right)$ is a weight-homogeneous polynomial with weight exponent $\mathbf{s}=\left(s_{1}, s_{2}, s_{3}, s_{4}\right)$ and weight degree $n$ if

$$
F\left(\alpha^{s_{1}} x_{1}, \alpha^{s_{2}} x_{2}, \alpha^{s_{3}} x_{3}, \alpha^{s_{4}} x_{4}\right)=\alpha^{n} F\left(x_{1}, x_{2}, x_{3}, x_{4}\right),
$$

If $\mathbf{s}=(1,1,1,1)$ then we say that $F$ is a homogeneous polynomial of degree $n$.

The following well-known proposition (easy to prove) reduces the study of the existence of analytic first integrals of a weight-homogeneous polynomial differential system (4) to the study of the existence of a weight-homogeneous polynomial first integrals.

Proposition 2. Let $H$ be an analytic function and let $H=\sum_{i} H_{i}$ be its decomposition into weight-homogeneous polynomials of weight degree $i$ with respect to the weight exponent $\mathbf{s}$. Then $H$ is an analytic first integral of the weight-homogeneous polynomial differential system (4) with weight exponent $\mathbf{s}$ if and only if each weighthomogeneous part $H_{i}$ is a first integral of system (4) for all $i$.

We have the Hamiltonian

$$
H=\frac{1}{2} \sum_{i=1}^{2} p_{i}^{2}+\frac{1}{2} a q_{1}^{2}\left(q_{1}+i q_{2}\right)^{2}+\frac{1}{4}\left(q_{1}^{2}+q_{2}^{2}\right)^{2},
$$

and its associated Hamiltonian system

$$
\begin{aligned}
& \dot{q}_{1}=p_{1}, \\
& \dot{q}_{2}=p_{2}, \\
& \dot{p}_{1}=-a q_{1}^{2}\left(q_{1}+i q_{2}\right)-a q_{1}\left(q_{1}+i q_{2}\right)^{2}-q_{1}\left(q_{1}^{2}+q_{2}^{2}\right), \\
& \dot{p}_{2}=-i a q_{1}^{2}\left(q_{1}+i q_{2}\right)-q_{2}\left(q_{1}^{2}+q_{2}^{2}\right) .
\end{aligned}
$$

By the change of variables $w_{1}=q_{1}+i q_{2}, w_{2}=q_{1}-i q_{2} v_{1}=p_{1}+i p_{2}, v_{2}=p_{1}-i p_{2}$ the differential system (5) becomes

$$
\begin{aligned}
& \dot{w}_{1}=v_{1}, \\
& \dot{w}_{2}=v_{2}, \\
& \dot{v}_{1}=-\frac{1}{2} w_{1}^{2}\left[(a+2) w_{2}+a w_{1}\right], \\
& \dot{v}_{2}=-\frac{1}{2} w_{1}\left[(a+2) w_{2}^{2}+a\left(2 w_{1}^{2}+3 w_{1} w_{2}\right)\right] .
\end{aligned}
$$

It has the first integral

$$
\bar{H}=4 v_{1} v_{2}+w_{1}^{2}\left[2 w_{2}^{2}+a\left(w_{1}+w_{2}\right)^{2}\right] .
$$

It is easy to check that system (6) is a weight-homogeneous polynomial differential system with weight exponent $(1,1,2,2)$ and weight degree 2 . Therefore, in view of Proposition 2 to study the existence of a second independent analytic first integral of system (6) it is enough to study the existence of a weight-homogeneous polynomial first integral of system (6) with weight exponent $(1,1,2,2)$. 


\section{Proof of the MAin Result}

Before going into the technicalities of the proof of our main result (Theorem 1), we would like to highlight the main idea behind it. First, we shall restrict system (6) to the zero level of the first integral $\bar{H}$, which is a polynomial function. The restriction to this level set gives rise to a non-trivial rational first integral $\bar{F}$ of the restricted system. To be more precise $\bar{F}\left(w_{1}, w_{2}, v_{2}\right) \in C\left(w_{1}, w_{2}, v_{2}\right)$ is a Laurent polynomial with respect to $v_{2}$, i.e., $\bar{F}\left(w_{1}, w_{2}, v_{2}\right) \in C\left[w_{1}, w_{2}\right]\left[v_{2}^{-1}, v_{2}\right]$. So, it can be written in the following form

$$
\bar{F}=\sum_{i=-k}^{m} g_{i}\left(w_{1}, w_{2}\right) v_{2}^{i}, \quad g_{m}\left(w_{1}, w_{2}\right) \neq 0
$$

and $g_{m+j}\left(w_{1}, w_{2}\right)=0$ for $j \geq 1$. We claim that knowing the two highest order terms of $g_{i}\left(w_{1}, w_{2}\right)$ with respect to $w_{2}$ for $-k+2 \leq i \leq m$, we achieve a contradiction considering the terms $g_{-k}\left(w_{1}, w_{2}\right)$ and $g_{-k+1}\left(w_{1}, w_{2}\right)$.

From sections 1 and 2 it follows that for proving Theorem 1 it is sufficient to show that if $a(a+2) \neq 0$, then system (6) has no weight-homogeneous polynomial first integrals with weight exponent $(1,1,2,2)$. Let $F=F\left(w_{1}, w_{2}, v_{1}, v_{2}\right) \in$ $\mathbb{C}\left[w_{1}, w_{2}, v_{1}, v_{2}\right]$ be a weight-homogeneous polynomial first integral of system (6) with weight exponent $(1,1,2,2)$ and weight degree $n \geq 1$. For each $n \geq 1$, we can express it as

$$
F=\sum_{l_{1}+l_{2}+2 l_{3}+2 l_{4}=n} F_{l_{1}, l_{2}, l_{3}, l_{4}} w_{1}^{l_{1}} w_{2}^{l_{2}} v_{1}^{l_{3}} v_{2}^{l_{4}}
$$

The function $F$ cannot depend only on $w_{1}$ and $w_{2}$. Indeed, if $F=F\left(w_{1}, w_{2}\right)$ then from (6) we get

$$
v_{1} \frac{\partial F}{\partial w_{1}}+v_{2} \frac{\partial F}{\partial w_{2}}=0,
$$

and consequently $F$ is a constant. So $F$ depends on $v_{1}$ or $v_{2}$ and thus $n \geq 2$.

We study the first integral $F$ on the level $\bar{H}=0$ by eliminating, for example, $v_{1}$ as follows

$$
v_{1}=-\frac{w_{1}^{2}\left[2 w_{2}^{2}+a\left(w_{1}+w_{2}\right)^{2}\right]}{4 v_{2}} .
$$

Thus we end up with the following system

$$
\begin{aligned}
& \dot{w}_{1}=-\frac{w_{1}^{2}}{4 v_{2}}\left[a w_{1}\left(w_{1}+2 w_{2}\right)+(a+2) w_{2}^{2}\right] \\
& \dot{w}_{2}=v_{2} \\
& \dot{v}_{2}=-\frac{w_{1}}{2}\left[a w_{1}\left(2 w_{1}+3 w_{2}\right)+(a+2) w_{2}^{2}\right] .
\end{aligned}
$$

Note that the restriction of the polynomial first integral $F$ to the level set $\bar{H}=0$ can be written as

$$
\begin{aligned}
\bar{F} & =\sum_{l_{1}+l_{2}+2 l_{3}+2 l_{4}=n} \frac{(-1)^{l_{3}}}{4^{l_{3}}} \bar{F}_{l_{1}, l_{2}, l_{3}, l_{4}} w_{1}^{l_{1}} w_{2}^{l_{2}}\left[\frac{w_{1}^{2}\left(2 w_{2}^{2}+a\left(w_{1}+w_{2}\right)^{2}\right)}{v_{2}}\right]^{l_{3}} v_{2}^{l_{4}} \\
& :=\sum_{i=-[n / 2]}^{[n / 2]} \bar{F}_{i}\left(w_{1}, w_{2}\right) v_{2}^{i},
\end{aligned}
$$


where each $\bar{F}_{i}\left(w_{1}, w_{2}\right)$ is a homogeneous polynomial of weight degree $n-2 i$. As usual $[x]$ denotes the integer part function of $x$. Setting

$$
\begin{aligned}
& A=\frac{w_{1}}{2}\left[a w_{1}\left(2 w_{1}+3 w_{2}\right)+(a+2) w_{2}^{2}\right], \\
& B=\frac{w_{1}^{2}}{4}\left[a w_{1}\left(w_{1}+2 w_{2}\right)+(a+2) w_{2}^{2}\right],
\end{aligned}
$$

we have that $\bar{F}$ is a first integral of system (8) if and only if it satisfies

$$
-\frac{B}{v_{2}} \frac{\partial \bar{F}}{\partial w_{1}}+v_{2} \frac{\partial \bar{F}}{\partial w_{2}}-A \frac{\partial \bar{F}}{\partial v_{2}}=0 .
$$

Computing in (10) the different coefficients of $v_{2}^{j}$ with $-[n / 2]-1 \leq j \leq[n / 2]+1$, we conclude that $\bar{F}$ is a first integral of system (8) if and only if

$$
\begin{aligned}
& \frac{\partial \bar{F}_{i}}{\partial w_{2}}=0, \quad \text { for } i=[n / 2],[n / 2]-1, \\
& \frac{\partial \bar{F}_{i}}{\partial w_{2}}-B \frac{\partial \bar{F}_{i+2}}{\partial w_{1}}-(i+2) A \bar{F}_{i+2}=0, \quad \text { for } i=[n / 2]-2, \ldots,-[n / 2], \\
& B \frac{\partial \bar{F}_{i}}{\partial w_{1}}+i A \bar{F}_{i}=0, \quad \text { for } i=-[n / 2]+1,-[n / 2] .
\end{aligned}
$$

It is clear that we can express the function $\bar{F}$ given in (9) as

$$
\bar{F}=\sum_{i=-[n / 2]}^{m} \bar{F}_{i}\left(w_{1}, w_{2}\right) v_{2}^{i}, \quad \bar{F}_{m}\left(w_{1}, w_{2}\right) \neq 0,
$$

and such that $\bar{F}_{m+j}\left(w_{1}, w_{2}\right)=0$ for $j \geq 1$.

In the following lemma we calculate the two terms of highest degree in $w_{2}$ of the functions $F_{i}\left(w_{1}, w_{2}\right)$. In what follows l.o.t. $\left(w_{2}\right)$ will denote the lower order terms in the variable $w_{2}$.

Lemma 3. For $l \geq 1$ and $m-2 l \geq-[n / 2]$ we have

$$
\begin{aligned}
\bar{F}_{m} & =C_{0} w_{1}^{n-2 m}, \\
\bar{F}_{m-2 l} & =C_{l}^{n} C_{0} w_{2}^{3 l} w_{1}^{n-2 m+l}(a+2)^{l}+D_{l}^{n, m} C_{0} w_{2}^{3 l-1} w_{1}^{n-2 m+l+1} a(a+2)^{l-1}+\text { l.o.t. }\left(w_{2}\right),
\end{aligned}
$$

where $C_{0} \neq 0$. Moreover the coefficients of the above polynomials are

$$
\begin{aligned}
C_{l}^{n} & =\prod_{k=1}^{l} \frac{n-3 k+3}{12 k}, \\
D_{l}^{n, m} & =\sum_{i=1}^{l} \frac{n+m-5 i+5}{2(3 i-1)} \prod_{j=i+1}^{l} \frac{n-3 j+4}{4(3 j-1)} \prod_{r=1}^{i-1} \frac{n-3 r+3}{12 r} .
\end{aligned}
$$

We note that $\bar{F}_{m-2 l}$ are the homogeneous polynomial of weight degree $n-2 m+4 l$.

Before proceeding with the proof of Lemma 3, we would like to highlight some basic facts about the expansion of the function of several variables. First, if we integrate an expansion with respect to, e.g., the variable $w_{2}$, then the additive constant depends generally on the remaining variables. Second, if we differentiate an expansion with respect to, e.g., variable $w_{2}$, then we can lose control on the leading term of expansion.

Proof of Lemma 3. Using (11) we get that

$$
\frac{\partial \bar{F}_{m}}{\partial w_{2}}=0 \text { that is } \quad \bar{F}_{m}=\bar{F}_{m}\left(w_{1}\right) .
$$


Since $\bar{F}_{m}$ has weight degree $n-2 m$ and we are assuming that is different from zero, we obtain $\bar{F}_{m}=C_{0} w_{1}^{n-2 m}$ with $C_{0} \neq 0$.

Now we use (11) with $i=m-2$, that is, we compute the coefficient of $v_{2}^{m-1}$ in (9). We get

$$
\begin{aligned}
\frac{\partial \bar{F}_{m-2}}{\partial w_{2}} & =B \frac{\partial \bar{F}_{m}}{\partial w_{1}}+m A \bar{F}_{m} \\
& =\frac{w_{1}^{2}}{4}\left[a w_{1}\left(w_{1}+2 w_{2}\right)+(a+2) w_{2}^{2}\right] C_{0}(n-2 m) w_{1}^{n-2 m-1} \\
& +\frac{m w_{1}}{2}\left[a w_{1}\left(2 w_{1}+3 w_{2}\right)+(a+2) w_{2}^{2}\right] C_{0} w_{1}^{n-2 m} \\
& =\frac{C_{0}}{4} w_{1}^{n-2 m+1}\left[n(a+2) w_{2}^{2}+2(n+m) a w_{1} w_{2}+\text { l.o.t. }\left(w_{2}\right)\right] .
\end{aligned}
$$

Integrating (13) in $w_{2}$ we get

$$
\begin{aligned}
\bar{F}_{m-2} & =\frac{C_{0}}{12} n(a+2) w_{1}^{n-2 m+1} w_{2}^{3}+\frac{C_{0}}{4}(n+m) a w_{1}^{n-2 m+2} w_{2}^{2}+\text { l.o.t. }\left(w_{2}\right) \\
& =C_{1}^{n} C_{0}(a+2) w_{1}^{n-2 m+1} w_{2}^{3}+C_{0} D_{1}^{n, m} a w_{1}^{n-2 m+2} w_{2}^{2}+\text { l.o.t. }\left(w_{2}\right) .
\end{aligned}
$$

This proves (3) with $l=1$ where $C_{1}^{n}=n / 12$ and $D_{1}^{n, m}=(n+m) / 4$.

Now we assume (3) is true for $l=1, \ldots, k$ and we will prove it for $k+1$ (assuming that $m-2(k+1) \notin\{-[n / 2]+1,-[n / 2]\})$. Computing the coefficient of $v_{2}^{m-2 k-1}$ in (9), that is considering (11) with $i=m-2 k-2$, we get

$$
\frac{\partial \bar{F}_{m-2(k+1)}}{\partial w_{2}}=B \frac{\partial \bar{F}_{m-2 k}}{\partial w_{1}}+(m-2 k) A \bar{F}_{m-2 k} .
$$

By the induction hypothesis we have

$$
\begin{aligned}
& \frac{\partial \bar{F}_{m-2(k+1)}}{\partial w_{2}} \\
& =\frac{w_{1}^{2}}{4}\left(a w_{1}\left(w_{1}+2 w_{2}\right)+(a+2) w_{2}^{2}\right)\left[C_{k}^{n} C_{0} w_{2}^{3 k}(n-2 m+k) w_{1}^{n-2 m+k-1}(a+2)^{k}\right. \\
& \left.+D_{k}^{n, m} C_{0} w_{2}^{3 k-1}(n-2 m+k+1) w_{1}^{n-2 m+k} a(a+2)^{k-1}+\text { l.o.t. }\left(w_{2}\right)\right] \\
& +\frac{(m-2 k) w_{1}}{2}\left(a w_{1}\left(2 w_{1}+3 w_{2}\right)+(a+2) w_{2}^{2}\right)\left[C_{k}^{n} C_{0} w_{2}^{3 k} w_{1}^{n-2 m+k}(a+2)^{k}\right. \\
& \left.+D_{k}^{n, m} C_{0} w_{2}^{3 k-1} w_{1}^{n-2 m+k+1} a(a+2)^{k-1}+\text { l.o.t. }\left(w_{2}\right)\right] \\
& =\tilde{C}_{k+1}^{n} C_{0} w_{2}^{3 k+2} w_{1}^{n-2 m+k+1}(a+2)^{k+1}+\tilde{D}_{k+1}^{n, m} C_{0} w_{2}^{3 k+1} w_{1}^{n-2 m+k+2} a(a+2)^{k} \\
& + \text { l.o.t. }\left(w_{2}\right),
\end{aligned}
$$

where

$$
\begin{aligned}
\tilde{C}_{k+1}^{n} & =\frac{n-3 k}{4} C_{k}^{n}=\frac{n-3(k+1)+3}{4} \prod_{i=1}^{k} \frac{n-3 i+3}{12 i}=3(k+1) \prod_{i=1}^{k+1} \frac{n-3 i+3}{12 i} \\
& =3(k+1) C_{k+1}^{n},
\end{aligned}
$$


and

$$
\begin{aligned}
\tilde{D}_{k+1}^{n, m} & =\frac{n+m-5 k}{2} C_{k}^{n}+\frac{n-3 k+1}{4} D_{k}^{n, m} \\
& =\frac{n+m-5(k+1)+5}{2} \prod_{i=1}^{k} \frac{n-3 i+3}{12 i} \\
& +\frac{n-3(k+1)+4}{4} \sum_{i=1}^{k} \frac{n+m-5 i+5}{2(3 i-1)} \prod_{j=i+1}^{k} \frac{n-3 j+4}{4(3 j-1)} \prod_{r=1}^{i-1} \frac{n-3 r+3}{12 r} \\
& =(3(k+1)-1) \frac{n+m-5(k+1)+5}{2(3(k+1)-1)} \prod_{i=1}^{k} \frac{n-3 i+3}{12 i} \\
& +(3(k+1)-1) \sum_{i=1}^{k} \frac{n+m-5 i+5}{2(3 i-1)} \prod_{j=i+1}^{k+1} \frac{n-3 j+4}{4(3 j-1)} \prod_{r=1}^{i-1} \frac{n-3 r+3}{12 r} \\
& =(3(k+1)-1) D_{k+1}^{n, m} .
\end{aligned}
$$

Integrating (15) with respect to $w_{2}$ we have

$$
\begin{aligned}
\bar{F}_{n-2(k+1)} & =\frac{\tilde{C}_{k+1}^{n}}{3(k+1)} C_{0} w_{2}^{3(k+1)} w_{1}^{n-2 m+k+1}(a+2)^{k+1} \\
& +\frac{\tilde{D}_{k+1}^{n, m}}{3 k+2} C_{0} w_{2}^{3(k+1)-1} w_{1}^{n-2 m+k+2} a(a+2)^{k}+\text { l.o.t. }\left(w_{2}\right) \\
& =C_{k+1}^{n} C_{0} w_{2}^{3(k+1)} w_{1}^{n-2 m+k+1}(a+2)^{k+1} \\
& +D_{k+1}^{n, m} C_{0} w_{2}^{3(k+1)-1} w_{1}^{n-2 m+k+2} a(a+2)^{k}+\text { l.o.t. }\left(w_{2}\right) .
\end{aligned}
$$

This proves (3) for $k+1$.

Lemma 4. Assume that $n$ is a multiple of 3 . Then

(a) if $m \geq\left[\frac{n}{2}\right]-\frac{n}{3}+2$, then $D_{l}^{n, m} \neq 0$ for $l \geq n / 3+1$,

(b) if $m<\left[\frac{n}{2}\right]-\frac{n}{3}+2$, then $C_{l}^{n} \neq 0$ for $l \geq 1$.

Proof. First we prove statement (a). It follows from the proof of Lemma 3 that for $l \geq 1, D_{l}^{n, m}$ satisfies

$$
D_{l+1}^{n, m}=\frac{n+m-5 l}{2(3 l+2)} C_{l}^{n}+\frac{n-3 l+1}{4(3 l+2)} D_{l}^{n, m} .
$$

We note that from (12) $C_{l}^{n}=0$ if $l \geq n / 3+1$ and hence, for $l \geq n / 3+2$ we have from (16) that

$$
D_{l}^{n, m}=D_{n / 3+1}^{n, m} \prod_{i=n / 3+2}^{l} \frac{n-3 i+4}{4(3 i-1)} .
$$

In particular, since $n$ is a multiple of 3 , if we show that $D_{n / 3+1}^{n, m} \neq 0$ then from (17) we have that $D_{l}^{n, m} \neq 0$ for any $l \geq n / 3+1$. We recall that since $\left[\frac{n}{2}\right] \geq m \geq\left[\frac{n}{2}\right]-\frac{n}{3}+2$ and $n$ is a multiple of 3 , we have $n \geq 6$. In what follows we will show that $D_{n / 3+1}^{n, m} \neq 0$. We proceed by contradiction. Assume that $D_{n / 3+1}^{n, m}=0$. Then it follows from (16) that

$$
D_{n / 3}^{n, m}=\left(\frac{4 n}{3}-2 m\right) \prod_{k=1}^{n / 3} \frac{n-3 k+3}{12 k}=\frac{1}{4^{n / 3}}\left(\frac{4 n}{3}-2 m\right) .
$$


We rewrite (16) in the following way

$$
\begin{aligned}
D_{l}^{n, m} & =\frac{4(3 l+2)}{n-3 l+1}\left[D_{l+1}^{n, m}+\frac{5 l-n-m}{2(3 l+2)} C_{l}^{n}\right] \\
& =\frac{4(3 l+2)}{n-3 l+1} D_{l+1}^{n, m}+\frac{2(5 l-n-m)}{n-3 l+1} \prod_{j=1}^{l} \frac{n-3 j+3}{12 j} .
\end{aligned}
$$

We claim that given a sequence of the form $x_{M}=\bar{x}$ and for $k=M-1, \ldots, N$

$$
x_{k}=A_{k+1} x_{k+1}+f_{k+1},
$$

then

$$
x_{N}=\bar{x} \prod_{l=N+1}^{M} A_{l}+\sum_{l=N+1}^{M} f_{l} \prod_{j=N+1}^{l-1} A_{j} .
$$

Now we prove the claim by backwards induction. For $k=M-1$ we have $x_{M-1}=$ $A_{M} x_{M}+f_{M}=A_{M} \bar{x}+f_{M}$ that is equation (20) with $N=M-1$. Now we assume is true for $N=M-1, \ldots, L$ and we will prove it for $N=L-1$. By backwards induction we can write

$$
\begin{aligned}
x_{L-1} & =A_{L} x_{L}+f_{L}=A_{L} \bar{x} \prod_{l=L+1}^{M} A_{l}+A_{L} \sum_{l=L+1}^{M} f_{l} \prod_{j=L+1}^{l-1} A_{j}+f_{L} \\
& =\bar{x} \prod_{l=L}^{M} A_{l}+\sum_{l=L+1}^{M} f_{l} \prod_{j=L}^{l-1} A_{j}+f_{L}=\bar{x} \prod_{l=L}^{M} A_{l}+\sum_{l=L}^{M} f_{l} \prod_{j=L}^{l-1} A_{j},
\end{aligned}
$$

which is (20) for $N=L-1$. Therefore, it follows from (20) with $N=1, x_{1}=D_{1}^{n, m}$, $M=n / 3, \bar{x}=x_{M}=D_{n / 3}^{n, m}($ see $(18))$,

$$
A_{l}=\frac{4(3 l-1)}{n-3 l+4} \quad \text { and } \quad f_{l}=\frac{2(5 l-n-m-5)}{n-3 l+4} \prod_{j=1}^{l-1} \frac{n-3 j+3}{12 j}
$$

that

$$
\begin{aligned}
D_{1}^{n, m}= & \frac{1}{4^{n / 3}}\left(\frac{4 n}{3}-2 m\right) \prod_{l=2}^{n / 3} \frac{4(3 l-1)}{n-3 l+4} \\
& +\sum_{l=2}^{n / 3} \frac{2(5 l-n-m-5)}{n-3 l+4} \prod_{j=1}^{l-1} \frac{n-3 j+3}{12 j} \prod_{r=2}^{l-1} \frac{4(3 r-1)}{n-3 r+4} .
\end{aligned}
$$

Now we know from Lemma 3 that $D_{1}^{n, m}=(n+m) / 4$. In particular the function

$$
G_{n, m}=D_{1}^{n, m+1}-D_{1}^{n, m}=\frac{n+m+1}{4}-\frac{n+m}{4}=\frac{1}{4} \text {. }
$$

Hence $G_{n, m}$ does not depend on neither $n$ nor $m$ and is equal to the constant $1 / 4$. From (21) we have

$$
G_{n, m}=-\frac{2}{4^{n / 3}} \prod_{l=2}^{n / 3} \frac{4(3 l-1)}{n-3 l+4}-2 \sum_{l=2}^{n / 3} \frac{1}{n-3 l+4} \prod_{j=1}^{l-1} \frac{n-3 j+3}{12 j} \prod_{r=2}^{l-1} \frac{4(3 r-1)}{n-3 r+4} .
$$

In particular $G_{n, m}$ given in (22) must be equal to $1 / 4$ for any value of $n$. When $n=6$ we get from $(22)$ that $G_{6, m}=-7 / 8 \neq 1 / 4$, a contradiction. This concludes the proof of the statement (a).

Now we prove the statement (b). Note that $C_{l}^{n}=0$ if $l \geq(n+3) / 3$. So we shall prove that $l<(n+3) / 3$. Since $m-2 l \geq-[n / 2]$ and $m<\left[\frac{n}{2}\right]-\frac{n}{3}+2$, we have that $2 l<[n / 2]-n / 3+2$ which concludes the proof. 
Proof of Theorem 1. We will show that $\bar{F}=0$. In fact we shall see that $\bar{F}_{i}=0$ for $i=-[n / 2], \ldots,[n / 2]$, and consequently $\bar{F}=0$. We note that by Lemma 3 if $n$ is not a multiple of 3 , then $C_{l}^{n} \neq 0$. On the other hand it follows from Lemma 4 that if $n$ is a multiple of 3 and if $m \geq\left[\frac{n}{2}\right]-\frac{n}{3}+2$ then $D_{l}^{n, m} \neq 0$ for $l \geq n / 3+1$; and if $m<\left[\frac{n}{2}\right]-\frac{n}{3}+2$, then $C_{l}^{n} \neq 0$ for $l \geq 1$.

Applying Lemma 3 and using (11) for $i=-[n / 2]$, we get

$$
\begin{aligned}
0= & B \frac{\partial \bar{F}_{-[n / 2]}}{\partial w_{1}}-[n / 2] A \bar{F}_{-[n / 2]} \\
= & 3(l+1) C_{l+1}^{n} C_{0} w_{2}^{3 l+2} w_{1}^{n-2 m+l+1}(a+2)^{l+1}+ \\
& (3 l+2) D_{l+1}^{n, m} C_{0} w_{2}^{3 l+1} w_{1}^{n-2 m+l+2} a(a+2)^{l}+\text { l.o.t. }\left(w_{2}\right) .
\end{aligned}
$$

Again applying Lemma 3 and using (11) for $i=-[n / 2]+1$, we have

$$
\begin{aligned}
0= & B \frac{\partial \bar{F}_{-[n / 2]+1}}{\partial w_{1}}+(-[n / 2]+1) A \bar{F}_{-[n / 2]+1} \\
= & 3(l+1) C_{l+1}^{n} C_{0} w_{2}^{3 l+2} w_{1}^{n-2 m+l+1}(a+2)^{l+1}+ \\
& (3 l+2) D_{l+1}^{n, m} C_{0} w_{2}^{3 l+1} w_{1}^{n-2 m+l+2} a(a+2)^{l}+\text { l.o.t. }\left(w_{2}\right) .
\end{aligned}
$$

Since $C_{0} a(a+2) \neq 0$, we obtain that $C_{l+1}^{n}=D_{l+1}^{n, m}=0$. But $C_{l}^{n} \neq 0$ if $n$ is not a multiple of 3 , or $n$ is a multiple of 3 and $m<[n / 2]-n / 3+2$, we have that $n$ is a multiple of 3 and $m \geq[n / 2]-n / 3+2$. Therefore, we have a contradiction since by Lemma 4 in this case $D_{l+1}^{n, m} \neq 0$. This completes the proof of the theorem.

\section{ACKNOWLEDGEMENTS}

We thank Maria Przybylska for sending us some references, information about the conjecture and some good comments. We also thank the referee for the valuable comments on our manuscript which helped to improve the presentation of this paper.

The first two authors are supported by the grants MCYT/FEDER MTM 200803437 and Generalitat de Catalunya 2009SGR410. The first author also is supported by the ICREA Academia. The third author is partially supported by FCT through CAMGDS, Lisbon.

\section{REFERENCES}

[1] T. Bountis, H. Segur, and F. Vivaldi, Integrable Hamiltonian systems and the Painlevé property, Phys. Rev. A (3) 25 (1982), no. 3, 1257-1264.

[2] Y.F. Chang, M. Tabor, and J. Weiss, Analytic structure of the Hénon-Heiles Hamiltonian in integrable and nonintegrable regimes, J. Math. Phys. 23 (1982), no. 4, 531-538.

[3] A. Goriely, Integrability and nonintegrability of dynamical systems, Advanced Series in Nonlinear Dynamics, vol. 19, World Scientific Publishing Co. Inc., River Edge, NJ, 2001.

[4] B. Grammaticos, B. Dorizzi, and R. Padjen, Painlevé property and integrals of motion for the Hénon-Heiles system, Phys. Lett. A 89 (1982), no. 3, 111-113.

[5] L.S. Hall, A theory of exact and approximate configurational invariants, Phys. D 8 (1983), no. 1-2, 90-116.

[6] J. Hietarinta, A search for integrable two-dimensional Hamiltonian systems with polynomial potential, Phys. Lett. A 96 (1983), 273-278.

[7] _ Direct methods for the search of the second invariant, Phys. Rep. 147 (1987), 87-154.

[8] A.J. Maciejewski and M. Przybylska, All meromorphically integrable 2D Hamiltonian systems with homogeneous potential of degree 3, Phys. Lett. A 327 (2004), no. 5-6, 461-473.

[9] _ Darboux points and integrability of Hamiltonian systems with homogeneous polynomial potential, J. Math. Phys. 46 (2005), no. 6, 062901.

[10] J.J. Morales Ruiz, Differential Galois theory and non-integrability of Hamiltonian systems, Progress in Mathematics, vol. 179, Birkhäuser Verlag, Basel, 1999.

[11] A. Ramani, B. Dorizzi, and B. Grammaticos, Painlevé conjecture revisited, Phys. Rev. Lett. 49 (1982), no. 21, 1539-1541. 
1 Departament de Matemàtiques, Universitat Autònoma de Barcelona, 08193 Bellaterra, Barcelona, Spain

E-mail address: jllibre@mat.uab.cat, mahdi@mat.uab.cat

2 Mathematics Department, University of North Carolina at Charlotte, Charlotte, North Carolina 28223, USA

E-mail address: adam.mahdi@uncc.edu

3 Faculty of Applied Mathematics, AGH University of Science and Technology, AL. Mickiewicza 30, 30-059 Kraków, Poland

4 Departamento de Matemática, Instituto Superior Técnico, Av. Rovisco Pais 1049001, Lisboa, Portugal

E-mail address: cvalls@math.ist.utl.pt 